\title{
Male mating behavior ${ }^{\star}$
}

Maureen M. Barr ${ }^{\S}$, School of Pharmacy, University of WisconsinMadison, Madison, WI 53705 USA

L. Rene Garcia ${ }^{\S}$, Department of Biology, Texas A \& M University, College Station, TX 77843-3258 USA

\section{Table of Contents}

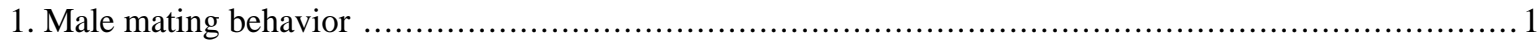

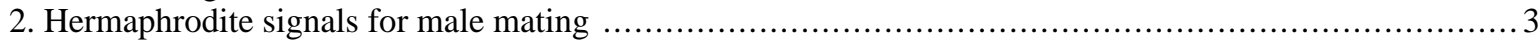

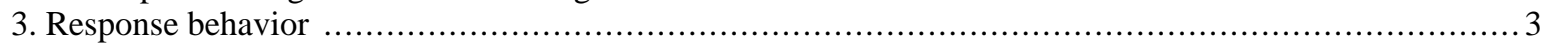

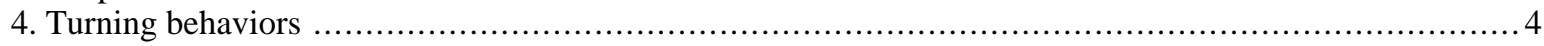

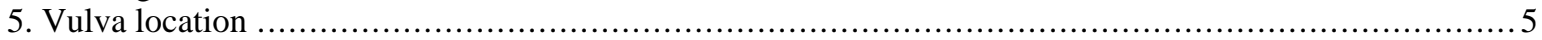

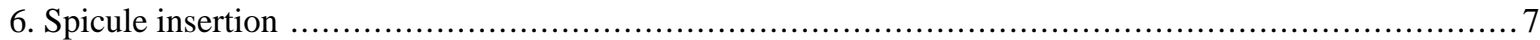

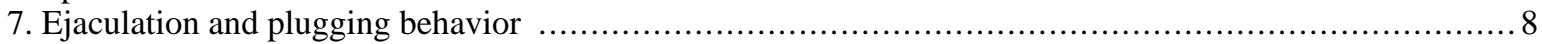

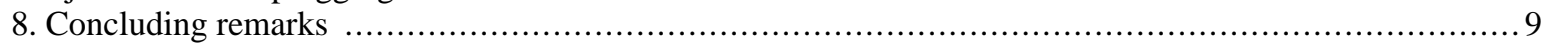

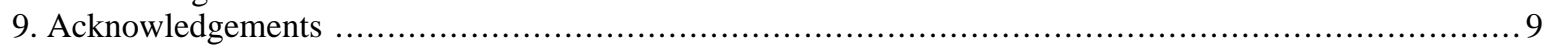

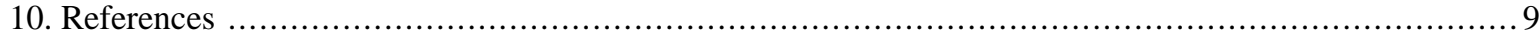

\begin{abstract}
Caenorhabditis elegans male mating provides an excellent opportunity to determine how sensory perception regulates behavior and motor programs. The male-specific nervous system and muscles are superimposed over the general nervous system and musculature. Genetic screens and genomic approaches have identified male-specific and male-enriched genes as well as non-sex specific molecules specialized for mating sub-behaviors. In this chapter, we discuss the cellular, genetic, and molecular basis for male mating behavior.
\end{abstract}

\section{Male mating behavior}

Copulation behavior is one of the more ancient social behaviors exhibited among metazoans. In C. elegans, the male performs most of the overt sensory and motor behaviors that occur during mating. Male mating behavior has been considered to be the most complex behavior in $C$. elegans; however although intricate, male mating behavior can be broken down into simpler sub-behaviors (Figure 1) that allow cellular and molecular dissection. In a

\footnotetext{
*Edited by Erik M. Jorgensen and Joshua M. Kaplan. Last revised March 10, 2005. Published June 19, 2006. This chapter should be cited as: Barr, M.M. and Garcia, L.R. Male mating behavior (June 19, 2006), WormBook, ed. The C. elegans Research Community, WormBook, doi/10.1895/wormbook.1.78.1, http://www.wormbook.org.

Copyright: (C) 2006 Maureen M. Barr and L. Rene Garcia. This is an open-access article distributed under the terms of the Creative Commons Attribution License, which permits unrestricted use, distribution, and reproduction in any medium, provided the original author and source are credited.

${ }^{\S}$ To whom correspondence should be addressed. E-mail: mmbarr@pharmacy.wisc.edu or rgarcia@mail.bio.tamu.edu
} 
stereotyped mating event (Movie 1), the male initially responds to hermaphrodite contact by placing his tail flush on her body; he begins moving backwards along her body until he reaches her head or tail, where he then turns via a sharp ventral coil. He continues backing until his tail contacts the vulva; at that region of the hermaphrodite, he stops moving, inserts his spicules, and ejaculates into the hermaphrodite uterus. Completion of all sub-behaviors is not mandatory for successful copulation. For example, if the initial contact is on the ventral side of the hermaphrodite, the male may immediately locate the vulva, insert his spicules, and ejaculate without evoking turning behavior. In this chapter, each step in the male-specific behavioral program is described at the levels of behavioral observation, anatomical and cellular requirements, and molecular genetic mechanisms. Neurons and genes required for male mating sub-steps are listed in Tables 1 and 2, respectively.

\section{Steps in male mating behavior}

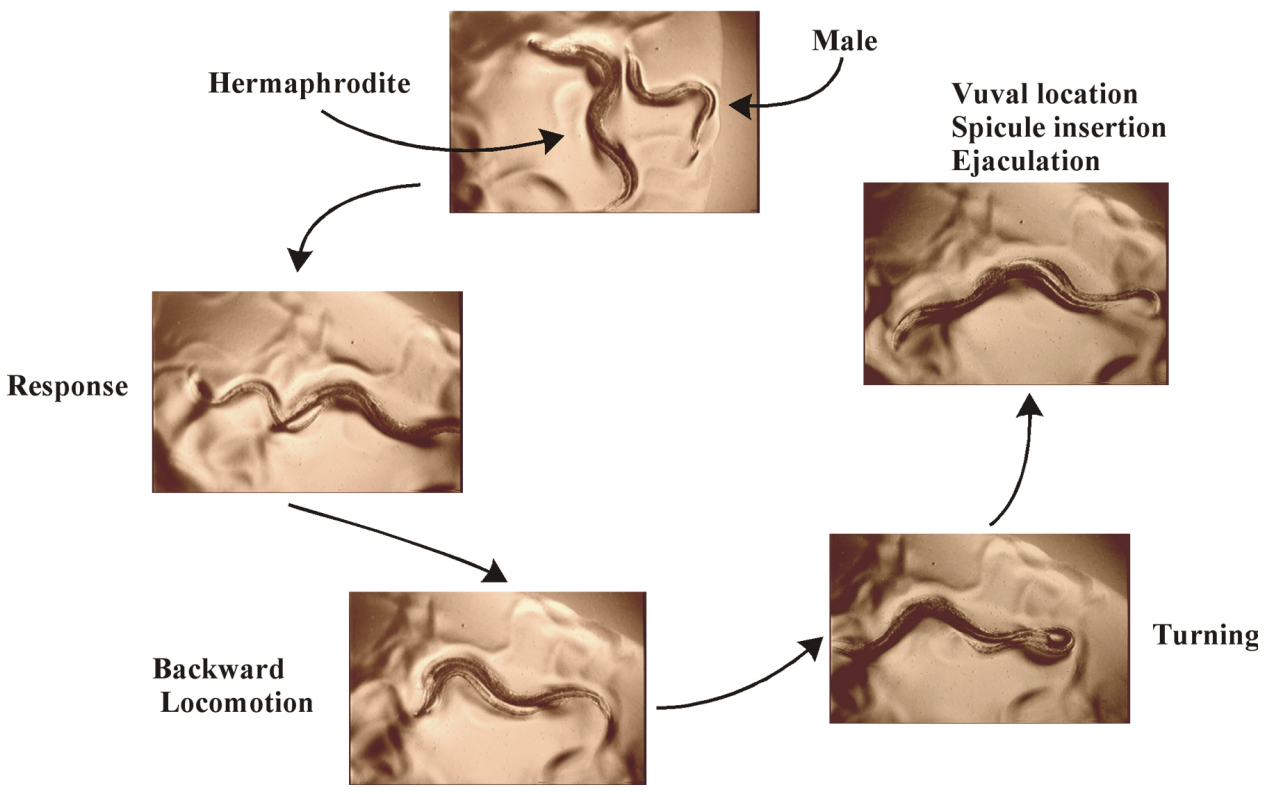

Figure 1. C. elegans male mating behavior.

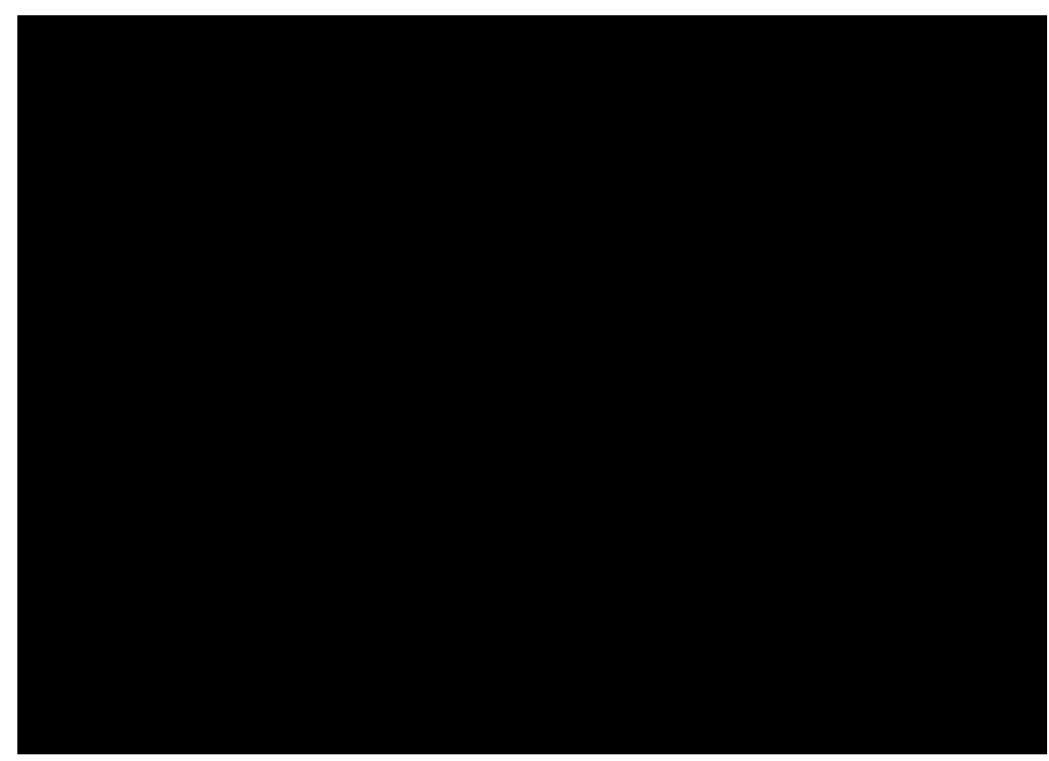

Movie 1. Wild-type C. elegans male mating behavior. 


\section{Hermaphrodite signals for male mating}

The hermaphrodite provides a combination of cues to the male before and during copulation. Prior to mating, the hermaphrodite provides sensory signals for attracting males. The adult hermaphrodite emits a diffusible mate finding cue that triggers males to increase reversal frequency (Simon and Sternberg, 2002). osm-5 and osm-6 cilium structure mutant males fail to respond to the mate-finding cue, indicating intact cilia are also required for this chemosensory behavior (Table 1). On a food source without hermaphrodites, males leave the food source and wander about their environment (Lipton et al., 2004). This "leaving behavior" is suppressed by the presence of an adult hermaphrodite, hinting at the existence of a short-range chemical cue or mechanosensory input. Nutritional status of males and the reproductive system of both males and hermaphrodites regulates leaving (Lipton et al., 2004).

During mating behavior, chemosensation and mechanosensation of hermaphrodite-based cues are probably involved in response and vulva location behaviors (Barr and Sternberg, 1999). These hermaphrodite-base cues likely consist of a combination of cuticle composition, changes in body shape, and secreted ligands from orifices. Srf (surface antigenicity abnormal) hermaphrodites provide a poor response signal to wild-type males, suggesting that a surface protein expressed on the cuticle provides a short-range signal (Wang and Barr, 2003). The hermaphrodite's vulva also provides cues to the male (Liu and Sternberg, 1995), some data suggests that the vulva location signal may consist of mechanosensory (a characteristic shape) and/or chemosensory (a combination of cell-specific chemicals) information, (Barr and Sternberg, 1998). A male also senses the end of the hermaphrodite body and executes a sharp ventral coil at the mate's head or tail, suggesting that his tail can sense a tapering of the hermaphrodite's body. The hermaphrodite's uterus may signal male sperm release, but the nature of this signal is unknown (Liu, 1996).

\section{Response behavior}

Male response behavior is initiated when sensory neurons located in the rays of his tail contact a potential mate. The male stops forward locomotion, presses the ventral side of his tail against his partner's body, and begins moving backward, scanning his partner's vulva. The bilateral pairs of sensory rays of the male tail, numbered 1 (anterior) to 9 (posterior) mediate response and turning behavior (Figure 2). Each ray is composed of a single structural cell and 2 sensory neurons $\mathrm{RnA}$ and $\mathrm{RnB}(\mathrm{n}=$ the ray number; for development of rays, see Male development). The dendritic processes of $\mathrm{RnA}$ and $\mathrm{RnB}$ extend down the length of each ray and terminate in exposed ciliated sensory endings (except R6; Sulston et al., 1980). Dorsally positioned rays $(1,5,7)$ are required for response to dorsal contact. Response to ventral contact requires the ventrally positioned rays $(2,4,8)$ but the ventral mating organs of the hook, p.c.s., and spicules also detect ventral contact (Liu and Sternberg, 1995).

Males with severe defects in all sensory neuron cilia, such as the mutants osm-1, osm-5, osm-6, and che-3, exhibit pleiotropic male mating defects in response, vulva location, and ejaculation (Barr and Sternberg, 1999; Qin et al., 2001). The only ciliated cells in C. elegans are chemosensory and mechanosensory neurons (White et al., 1986). The male has 46 predicted ciliated sensory neurons in his tail and 4 in his head (Sulston et al., 1980). osm-5::gfp and osm-6::GFP are expressed exclusively in ciliated neurons, with male-specific expression in four CEM head neurons, the A- and B-type neurons of the hook and rays, post cloacal sensillae (p.c.s.), and copulatory spicules (Collet et al., 1998; Qin et al., 2001). A chemosensory role for the male-specific CEMs has been proposed but not empirically demonstrated.

Response behavior requires the lov-1-encoded polycystin-1 11-transmembrane spanning receptor, pkd-2encoded polycystin-2 TRP (transient receptor potential) channel, and the klp- 6 encoded kinesin-3 family member (Barr et al., 2001; Barr and Sternberg, 1999; Peden and Barr, 2005). lov-1, pkd-2, and klp-6 are expressed in the male-specific sensory neurons in the head (the CEMs), rays (RnBs 1-9 except 6), and hook (HOB). klp-6 is also expressed in IL2 neurons in both hermaphrodites and males. The $l o v-1$ and $p k d-2$ proteins localize to cilia, hinting at a role in sensory reception. $k l p-6::$ GFP is distributed throughout the sensory neuron, including axon, cell body (not nucleus), dendrite and cilium. The putative cargo binding domain of klp- 6 is sufficient to target the kinesin to cilia (Peden and Barr, 2005). klp-6 is required for PKD-2::GFP ciliary localization, consistent with a cargo-motor relationship between lov-1 and pkd-2 with klp-6 (Peden and Barr, 2005). Kinesins typically use adaptors to link cargo to motor, but whether this interaction between the polycystins and klp-6 is direct or indirect remains to be determined. 
A

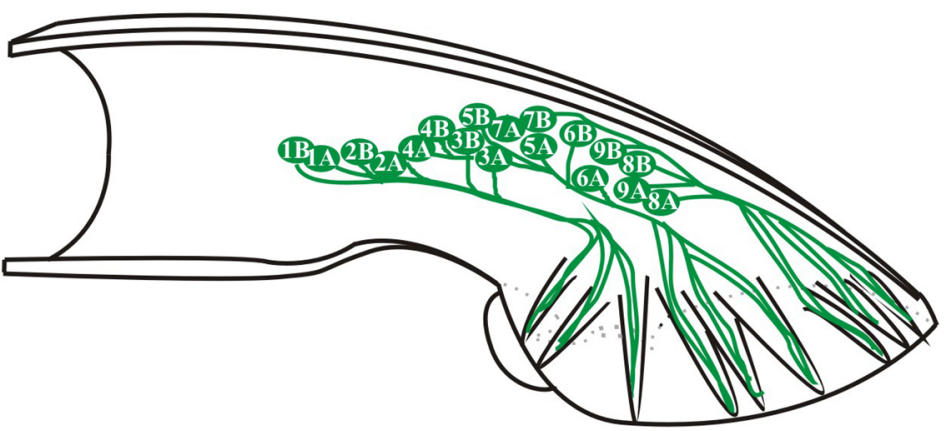

B

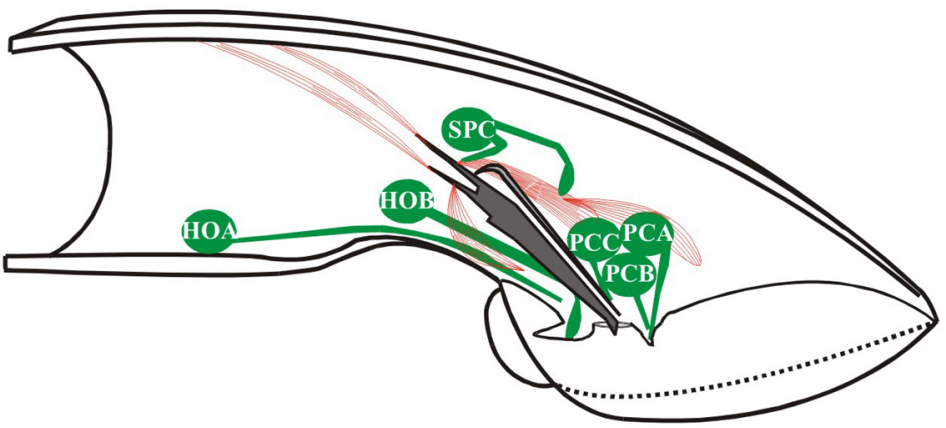

Figure 2. Male sensory neurons associated with mating behavior. Cartoon adapted from Sulston et al. (1980) depicting the positions of ray neurons (A) and hook and postcloacal sensilla (p.c.s.) and SPC neurons (B). In panel A, the male tail has 9 bilaterally arranged rays numbered 1-9 anterior to posterior, (only one side is shown). Each ray contains the sensory dendritic process of an A type neuron and a B type neuron. Ray neurons are labeled according to what neuronal type they are, and with which rays they are associated. In panel B, sensory dendritic processes of the HOA and HOB neurons (asymmetrically located on the left side of the animal) and the p.c.s. neurons (a left/right bilateral set of three neurons) are associated with the cloacal opening. The SPC proprioceptive neuron is physically associated with the spicule protractor muscles.

\section{Turning behaviors}

After responding, the male backs until encountering and turning at the mate's head or tail. Turning behavior involves sensory input from the ray neurons and locomotory behaviors mediated by the male specific CP ventral cord motor neurons and EF interneurons. Ablation studies indicate that the $\mathrm{CP}$ neurons and 3 posterior-most rays (Rays 7-9) are essential for turning behavior (Liu and Sternberg, 1995; Loer and Kenyon, 1993). The CP neurons synapse onto the male-specific diagonal muscles, which are responsible for flexing the tail ventrally or dorsally (White, 1988).

Turning behavior is mediated by the neurotransmitters serotonin and dopamine (Loer and Kenyon, 1993). Exogenously applied serotonin induces ventral male tail curling, similar to that observed during mating (Loer and Kenyon, 1993). R1B, R3B, and R9B and male-specific CP motoneurons contain serotonin (Loer and Kenyon, 1993; Lints et al., 2004). The dopamine containing R5A, R7A, and R9A rays are required for the timing of sharp ventral turns, with ablated or dopamine deficient cat-2 mutant males exhibiting sloppy turns (Lints and Emmons, 1999; Sulston et al., 1980; Sulston and Horvitz, 1977). Neuropeptides may perform specialized tasks in male mating behaviors (T. Liu and M. Barr, unpublished). Mutants defective in neuropeptide biosynthesis or function exhibit an abnormal turning phenotype. 
Table 1. Neurons required for male mating behavior

\begin{tabular}{|l|l|}
\hline Behavioral sub-step & Neuron(s) \\
\hline Response & Rays 1-6 (Liu and Sternberg, 1995), RnBs (Barr and Sternberg, 1999) \\
\hline Turning & CP neurons, Rays 7-9 (Liu and Sternberg, 1995; Loer and Kenyon, 1993) \\
\hline Vulva location & HOA and HOB (Liu and Sternberg, 1995) \\
\hline General & PCA, PCB, and PCC (Liu and Sternberg, 1995) \\
\hline Specific & \multicolumn{2}{|l}{} \\
\hline Spicule insertion & Hook and p.c.s. neurons (Garcia et al., 2001) \\
\hline Periodic prodding & SPC, PCB, and PCC (Garcia et al., 2001; Liu and Sternberg, 1995) \\
\hline Sustained protraction & SPV (Liu and Sternberg, 1995) \\
\hline Ejaculation-inhibition &
\end{tabular}

\section{Vulva location}

When backing along the hermaphrodite's ventral side, the male encounters the vulva (representing approximately $1 / 200^{\text {th }}$ of the ventral length). Vulva location behavior is complex: the male stops at the vulva, coordinates his movements to the hermaphrodite's, and positions his tail precisely over the vulva so that he may insert his spicules and ejaculate. General vulva location (stopping) requires the hook sensillum while precise vulva location (coordinating movement and tail positioning) requires the p.c.s. and spicules (Liu and Sternberg, 1995; Loer et al., 1999). The single-cell derived hook structure houses the hook sensillum, consisting of two sensory neurons (HOA and HOB) and two support cells (Sulston et al., 1980). The HOB neuron may be chemosensory by ultrastructural criteria (its ending opens externally through a socket in the hook) while HOA may be mechanosensory (its rootlet is striated and its ciliated dendritic ending terminates before opening). The anatomy of $\mathrm{HOA}$ and $\mathrm{HOB}$ is very similar to $\mathrm{RnA}$ and $\mathrm{RnB}$ : the B-type cilium is exposed to the environment and positioned next to the embedded A-type cilium. HOA and HOB form multiple chemical synapses and electrical junctions (Sulston et al., 1980), indicating extensive cross talk between the two hook sensory neurons. HOB also has pre- and post-synaptic connections with several male-specific interneurons and motor neurons (Wormatlas male wiring project). HOA has a pre-synaptic interaction with the ventral cord motor neuron VD13. The p.c.s. are arranged as a bilateral pair, each sensillum composed of three neurons (PCA, PCB, PCC) and three support cells (Sulston et al., 1980).

lov-1, pkd-2, and klp-6 mutants are also Lov (location of vulva) defective (Barr et al., 2001; Barr and Sternberg, 1999; Peden and Barr, 2005; Movie 2). LOV-1::GFP and PKD-2::GFP localize to the cilium of HOB, suggesting that the two act as a sensory receptor/channel complex. However, lov- 1 and $p k d-2$ mutants are able to successfully locate the vulva in $25 \%$ of contacts (in contrast to $0 \%$ of HOB ablated animals), hinting at the existence of another sensory pathway required for vulva location. klp-6 mutants exhibit a $40 \%$ vulva location efficiency, suggesting that the klp-6 kinesin may have negative regulatory cargoes in addition to lov-1 and pkd-2 (Peden and Barr, 2005). 


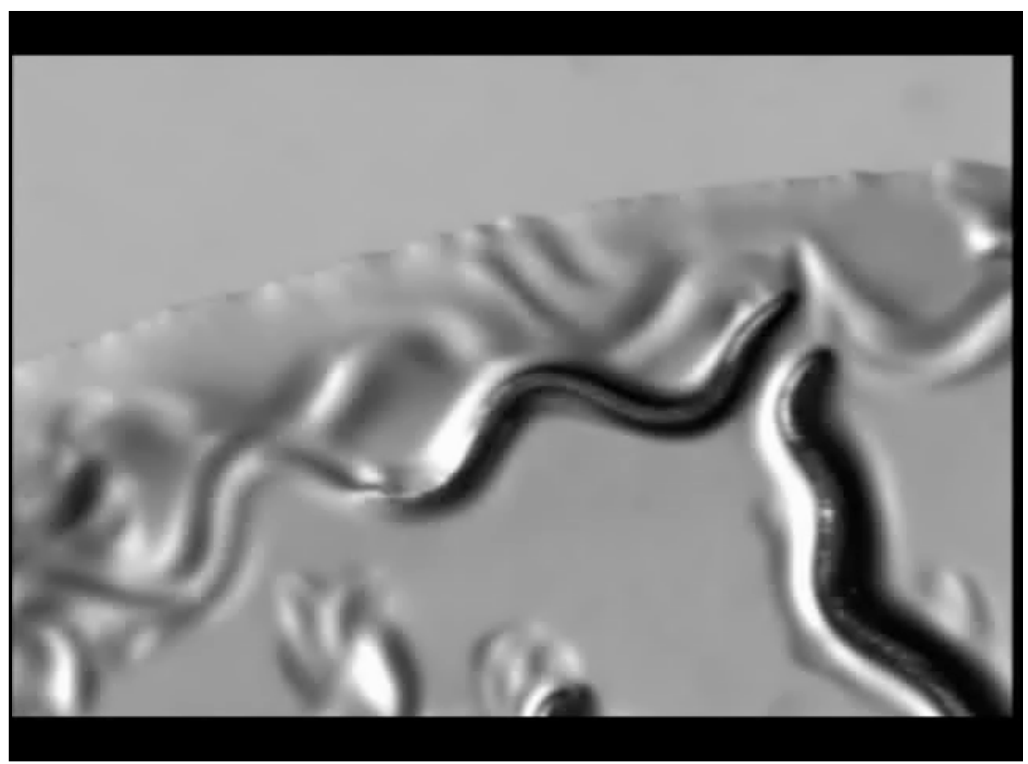

Movie 2. pkd-2 mutants are response and Lov defective.

Table 2. Genes required for male mating behavior

\begin{tabular}{|c|c|c|c|}
\hline Behavior & $\begin{array}{l}\text { Gene as determined by } \\
\text { behavioral assay }\end{array}$ & Gene product & Behavioral reference \\
\hline Mate-finding & $\begin{array}{l}o s m-5 \\
\text { osm-6 }\end{array}$ & $\begin{array}{l}\text { Cilium structure gene } \\
\text { Cilium structure gene }\end{array}$ & Simon and Sternberg, 2002 \\
\hline Drive & $\begin{array}{l}\text { unc-77 } \\
\text { tph-1 } \\
\text { daf-2 } \\
\text { glp-1 } \\
\text { spe-26 }\end{array}$ & $\begin{array}{l}\text { Unknown } \\
\text { Serotonin biosynthesis } \\
\text { Insulin receptor } \\
\text { Germ cell development } \\
\text { Germ cell development }\end{array}$ & $\begin{array}{l}\text { Emmons and Lipton, } 2003 \\
\text { Lipton et al., } 2004\end{array}$ \\
\hline Response & $\begin{array}{l}\text { osm-5 } \\
\text { lov-1 } \\
\text { pkd-2 } \\
\text { klp-6 }\end{array}$ & $\begin{array}{l}\text { Cilium structure genes } \\
\text { Membrane receptor } \\
\text { TRP channel } \\
\text { Kinesin-3 family member }\end{array}$ & $\begin{array}{l}\text { Barr and Sternberg, } 1999 \\
\text { Barr et al., } 2001 \\
\text { Peden and Barr, } 2005\end{array}$ \\
\hline Turning & $\begin{array}{l}\text { cat }-2 \\
\text { cat-1 } \\
\text { cat-4 } \\
\text { bas-1 }\end{array}$ & $\begin{array}{l}\text { Tyrosine hydroxylase } \\
\text { Vesicular monoamine transporter } \\
\text { GTP cyclohydrolase I } \\
\text { aromatic amino acid } \\
\text { decarboxylase }\end{array}$ & $\begin{array}{l}\text { Lints and Emmons, } 1999 \\
\text { Loer and Kenyon, } 1993\end{array}$ \\
\hline Vulva location & $\begin{array}{l}\text { osm-5 } \\
l o v-1 \\
p k d-2 \\
k l p-6\end{array}$ & $\begin{array}{l}\text { Cilium structure gene } \\
\text { Membrane receptor } \\
\text { TRP channel } \\
\text { Kinesin-3 family member }\end{array}$ & $\begin{array}{l}\text { Barr and Sternberg, } 1999 \\
\text { Barr et al., } 2001 \\
\text { Peden and Barr, } 2005\end{array}$ \\
\hline Spicule insertion & $\begin{array}{l}\text { egl-19 } \\
\text { unc-29 } \\
\text { unc-38 }\end{array}$ & $\begin{array}{l}\text { L-type voltage-gated } \mathrm{Ca}^{2+} \\
\text { channel } \\
\text { Nicotinic acetylcholine receptor } \\
\text { Nicotinic acetylcholine receptor }\end{array}$ & Garcia et al., 2001 \\
\hline
\end{tabular}




\begin{tabular}{|c|c|c|c|}
\hline Behavior & $\begin{array}{l}\text { Gene as determined by } \\
\text { behavioral assay }\end{array}$ & Gene product & Behavioral reference \\
\hline & $\begin{array}{l}\text { unc-68 } \\
\text { egl-30 } \\
\text { unc-103 } \\
\text { goa-1 }\end{array}$ & 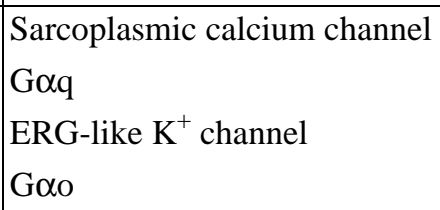 & $\begin{array}{l}\text { Garcia and Sternberg, } 2003 \\
\text { Mendel et al., } 1995\end{array}$ \\
\hline Sperm transfer & $\begin{array}{l}\text { osm-5 } \\
\text { plg-1 }\end{array}$ & $\begin{array}{l}\text { Cilium structure gene } \\
\text { Plug formation }\end{array}$ & $\begin{array}{l}\text { Qin et al., } 2001 \\
\text { Hodgkin and Doniach, } 1997 .\end{array}$ \\
\hline
\end{tabular}

\section{Spicule insertion}

Spicule insertion behavior initiates when the male cloaca contacts the vulva. The purpose of this behavior is to clasp the male tail to the vulva and pry apart the vulval lips so that sperm flows into the uterus. Spicule insertion behavior is facilitated by the coordinate actions of male-specific neurons and sex muscles located in the male tail (Figure 2 and Figure 3). The male contains a bilateral set of spicules; each contains portions of two sheath cells, four socket cells, and the sensory dendrites of the SPV and SPD neurons, all encased within a sclerotized cuticle (Sulston et al., 1980). The SPV and SPD cell bodies reside outside the spicules, but their dendrites run through the spicules and their sensory endings are exposed to the environment at the spicule tips. Associated with each spicule are protractor and retractor muscles that control spicule movement; shortening of the protractors causes the spicules to extrude from the tail, whereas shortening of the retractors withdraws them back. Also connected to both left and right dorsal protractors is an accessory muscle that is derived from the anal depressor muscle; however, under laboratory conditions, this accessory is not essential for mating (Garcia et al., 2001).

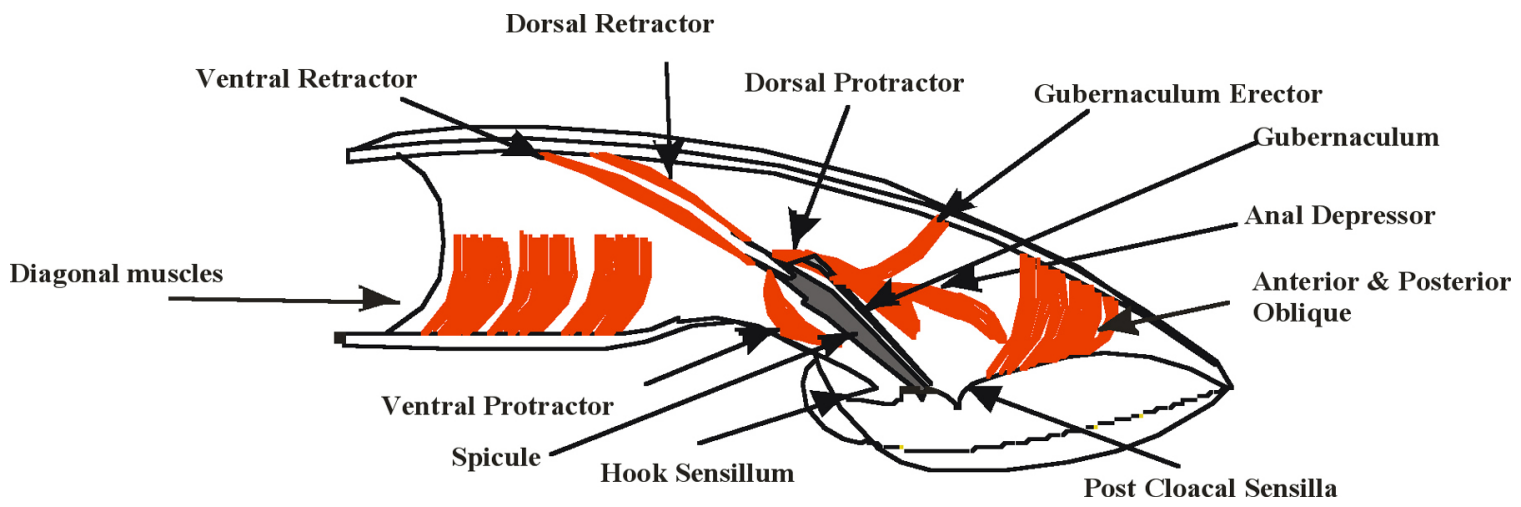

Figure 3. Male sex muscles associated with mating behavior. Cartoon adapted from Sulston et al. (1980). Cutaway view of the right half of the male tail. Muscles are represented in red, the sclerotized right spicule is represented in gray.

During vulval contact, the protractors contract $\sim 7$ to 11 times a second, causing the spicule tips to prod the vulva with a repetitive thrusting motion (Figure 3). A slight shift of the cloaca from the vulva results in the termination of this prodding behavior. The hook and p.c.s. neurons, in addition to sensing the vulva, initiate the protractors to undergo the periodic contractions (Garcia et al., 2001). Stimulation of the spicule muscles by the hook and p.c.s. is indirect since neither innervates the spicule muscles. The hook sensillum neuron HOB has pre- and post-synaptic interactions as well as electrical junctions with the p.c.s. neurons and SPC motor neurons (Wormatlas male wiring project). In contrast, the p.c.s. neurons do synapse to other sex muscles in the male tail that are active during prodding behavior. The PCA neurons innervate the gubernaculum erector (see Wormatlas male wiring project), which controls the movements of the gubernaculum, a V- shaped thin sclerotized cuticular structure that has been proposed to guide the spicules out the male proctodeum (Sulston et al., 1980). In addition, PCA with PCB, and PCC innervate the left and right anterior and posterior oblique muscles. These muscles control dorsal and ventral bending of regions posterior of the cloacal opening (Wormatlas male wiring project). During prodding behavior, the gubernaculum twitches with the spicules while the male presses his cloaca and posterior regions of his tail against the vulva; presumably, the male uses these neurons to execute gubernaculum movements and regulate the posture of his posterior tail during spicule insertion. No obvious electrical connections between the oblique and 
gubernaculum muscles to the protractors have been observed (Wormatlas male wiring project); therefore, the p.c.s. neurons may stimulate the protractor muscles indirectly.

During most mating encounters, the spicule tips will prod the vulva continuously until they partially penetrate, which then causes the protractors to contract completely so that the spicules extend through the vulva. After penetration, all spicule movements cease until ejaculation is completed. Sustained protractor contraction is triggered by the left and right SPC motor neurons (Garcia et al., 2001). These neurons make neuromuscular junctions to the dorsal and ventral protractor muscles, as well as the anal depressor muscle and the male gonad. They also have sensory endings that are physically attached to the dorsal protractors and the reorganized anal depressor via half desmosomes, suggesting that these cells may have proprioceptive functions. In addition, the SPC neurons also innervate the male gonad and make gap junctions and pre- and post-synaptic interactions with HOB and the p.c.s. neurons (Wormatlas male wiring project; Sulston et al., 1980). These connections emphasize that full spicule insertion behavior must be tightly coordinated with vulva location behavior and the subsequent sperm transfer step.

Agonists of acetylcholine (ACh) and inhibitors of ACh esterase such as aldicarb will induce spicule protraction, suggesting that $\mathrm{ACh}$ is the main excitatory neurotransmitter for this motor behavior. The endogenous sources of ACh that stimulate the protractors are the SPC, PCB, and PCC neurons, which is consistent with their function in triggering muscle contractions during prodding behavior and full spicule penetration. Levamisol, arecoline, and nicotine will directly induce the protractors to contract. In addition to activating different ACh receptors, these drugs also have differential requirements for intra- and extracellular calcium signaling. To induce spicule protraction, levamisol requires muscle-expressed unc-68-encoded ryanodine receptor calcium channels, whereas arecoline requires the muscle-expressed egl-19-encoded voltage-gated calcium channel; in contrast, nicotine requires both channels. The genetic requirements for these drugs suggest that the protractors may differentially use these calcium channels to execute rapid periodic contractions and sustained contraction. Mating observations of channel mutants suggest that the protractors use unc- 68 channels during prodding behavior and egl-19 channels for full spicule penetration (Garcia et al., 2001).

During prodding behavior, sustained protractor contraction is inhibited by the unc-103-encoded ERG-like $\mathrm{K}^{+}$ channel. Hermaphrodites and larval males containing unc-103(lf) mutations have no gross behavioral phenotypes, but adult males will protract their spicules completely in the absence of mating cues and during prodding behavior at the vulva (Garcia and Sternberg, 2003). unc-103 is expressed in many pharyngeal and nerve ring neurons, all cholinergic neurons in the ventral cord in both sexes, as well as the SPC, SPV, PCB, and PCC neurons (Gruninger et al., 2006). Presumably, expression in SPC, PCB, and PCC is required for regulating the proper timing of periodic and sustained contractions during mating.

\section{Ejaculation and plugging behavior}

Ejaculation occurs after spicule penetration, and lasts $\sim 4$ seconds. Little is known about sperm transfer, but direct gonadal innervation by SPC, PCB and PCC suggests that vulval contact and fully extended spicules may trigger this step (Wormatlas male wiring project). The SPV neurons have an interesting role in coordinating spicule penetration and ejaculation. Laser ablation of these neurons results in males that prematurely ejaculate during prodding behavior (Liu and Sternberg, 1995). The SPV neurons are gap-junctioned to the SPC neurons and may regulate their interactions with the gonad (Wormatlas male wiring project).

During ejaculation, the spicules remain inserted for about a minute. After spicule retraction, the male remains in contact with the vulva for a few more seconds (Liu and Sternberg, 1995). During this period, males of other Caenorhabditis species will generate a gelatinous plug (ranging from a thin film to a 100 micrometer diameter blob) on the vulva. N2 males cannot form a plug, but they still remain in contact with the hermaphrodite during this post-coital phase. Plugging ability is facilitated by the dominant plg-1(e2001) allele (Hodgkin and Doniach, 1997). This allele may facilitate the coagulation of some material in the seminal fluid. During ejaculation, a yellowish fluid is passed from the seminal vesicle and through the cloaca; in plugging strains, this fluid coagulates on the surface of the vulva, whereas it dissipates during N2 matings (Barker, 1994). In the laboratory, a copulation plug does not block the ability of a male to fertilize the plugged hermaphrodite (Hodgkin and Doniach, 1997). However, the plug can lengthen the time it takes a male to locate the vulva and insert its spicules (Barker, 1994). 


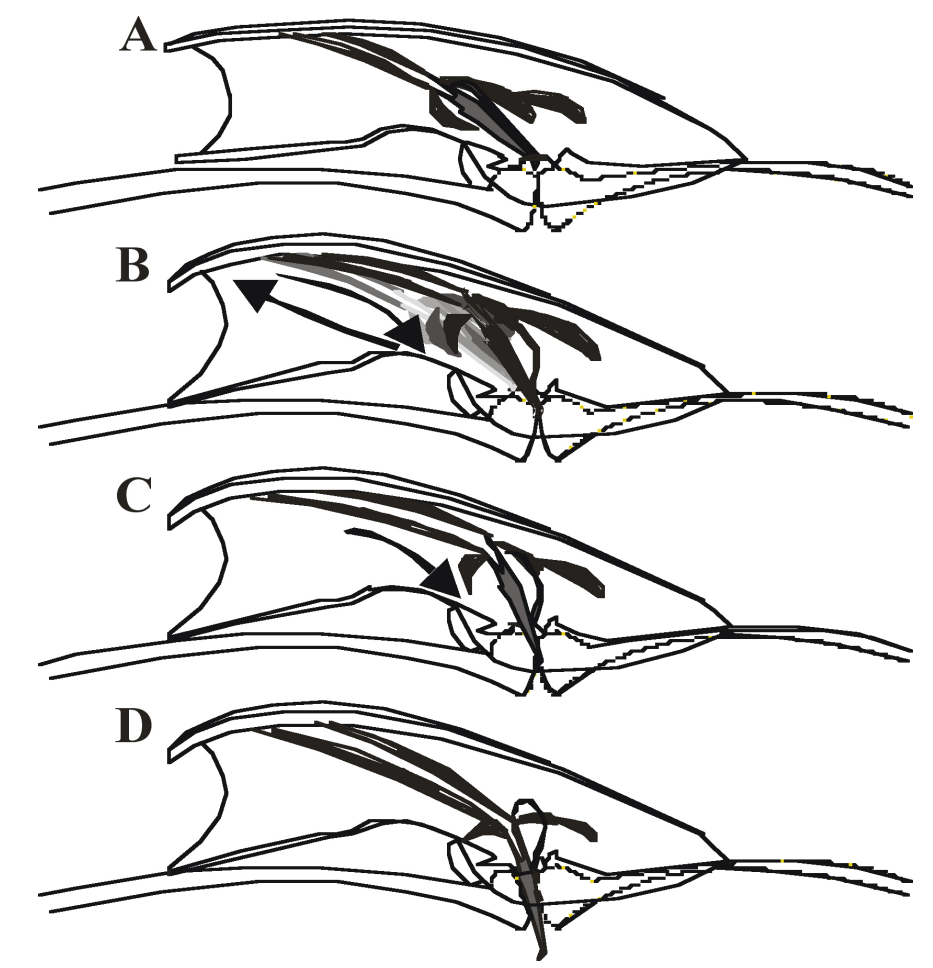

Figure 4. Steps during spicule insertion. Representation of the male tail over the hermaphrodite vulval region. (A) Contact with the hermaphrodite vulva initiates insertion behavior. (B) Spicules repeatedly prod the vulval slit. Arrows and variably shaded spicules denote repetitive shallow thrusting motions. (C) Partial penetration of the spicules signals prodding to stop and induces full insertion. Arrow denotes downward motion of the spicules. (D) Spicules stay inserted until ejaculation is over.

\section{Concluding remarks}

The diverse ways an animal responds to stimuli are shaped by the cellular components that form behavioral circuits, which in turn are regulated by the genes that are expressed in the circuit's specific neurons and muscles. Current studies of male mating behavior have provided insights on how an animal uses different sub-behaviors to perform an instinctive behavior. The current challenge is to determine the unique interactions between male-specific and general behavioral genes that allow neurons in the male to sense, integrate, and then translate signals into a physical output that ultimately allows him to accomplish an evolutionarily conserved task.

\section{Acknowledgements}

This research is sponsored by grants from the NIH (5R01DK059418 to M.M.B. and 1R01GM070431 to L.R.G.) and the PKD Foundation (to M.M.B.). L.R.G. is supported by the Searle Scholar Program.

\section{References}

Barker, D.M. (1994). Copulatory plugs and paternity assurance in the nematode Caenorhabditis elegans. Anim. Behav. 48, 147-156. Article

Barr, M.M., DeModena, J., Braun, D., Nguyen, C.Q., Hall, D.H., and Sternberg, P.W. (2001). The Caenorhabditis elegans autosomal dominant polycystic kidney disease gene homologs $l o v-1$ and $p k d-2$ act in the same pathway. Curr. Biol. 11, 1341-1346. Abstract Article

Barr, M.M., and Sternberg, P.W. (1998). Signals for C. elegans male mating behavior: A cellular and genetic approach to Lov (location of vulva). 1998 West Coast Worm Meeting.

Barr, M.M., and Sternberg, P.W. (1999). A polycystic kidney-disease gene homologue required for male mating behaviour in C. elegans. Nature 401, 386-389. Abstract Article 
Collet, J., Spike, C.A., Lundquist, E.A., Shaw, J.E., and Herman, R.K. (1998). Analysis of osm-6, a gene that affects sensory cilium structure and sensory neuron function in Caenorhabditis elegans. Genetics 148, 187-200. Abstract

Emmons, S.W., and Lipton, J. (2003). Genetic basis of male sexual behavior. J. Neurobiol. 54, 93-110. Abstract Article

Garcia, L.R., Mehta, P., and Sternberg, P.W. (2001). Regulation of distinct muscle behaviors controls the $C$. elegans male's copulatory spicules during mating. Cell 107, 777-788. Abstract Article

Garcia, L.R., and Sternberg, P.W. (2003). Caenorhabditis elegans UNC-103 ERG-like potassium channel regulates contractile behaviors of sex muscles in males before and during mating. J. Neurosci. 23, 2696-2705. Abstract

Gruninger, T.R., Gualberto, D.G., LeBoeuf, B., and Garcia, L.R. (2006). Integration of male mating and feeding behaviors in Caenorhabditis elegans. J. Neurosci. 26, 169-179. Abstract Article

Hodgkin, J., and Doniach, T. (1997). Natural variation and copulatory plug formation in Caenorhabditis elegans. Genetics 146, 149-164. Abstract

Lints, R., and Emmons, S.W. (1999). Patterning of dopaminergic neurotransmitter identity among Caenorhabditis elegans ray sensory neurons by a TGF $\beta$ family signaling pathway and a Hox gene. Development $126,5819-5831$. Abstract

Lints, R., Jia, L., Kim, K., Li, C., and Emmons, S.W. (2004). Axial patterning of C. elegans male sensilla identities by selector genes. Dev. Biol. 269, 137-151. Abstract Article

Lipton, J., Kleemann, G., Ghosh, R., Lints, R., and Emmons, S.W. (2004). Mate searching in Caenorhabditis elegans: a genetic model for sex drive in a simple invertebrate. J. Neurosci. 24, 7427-7434. Abstract Article

Liu, K.S. (1996), Ph.D. Thesis. California Institute of Technology, Pasadena.

Liu, K.S., and Sternberg, P.W. (1995). Sensory regulation of male mating behavior in Caenorhabditis elegans. Neuron 14, 79-89. Abstract Article

Loer, C.M., Davidson, B., and McKerrow, J. (1999). A phenylalanine hydroxylase gene from the nematode $C$. elegans is expressed in the hypodermis. J. Neurogenet. 13, 157-180. Abstract

Loer, C.M., and Kenyon, C.J. (1993). Serotonin-deficient mutants and male mating behavior in the nematode Caenorhabditis elegans. J. Neurosci. 13, 5407-5417. Abstract

Mendel, J.E., Korswagen, H.C., Liu, K.S., Hajdu-Cronin, Y.M., Simon, M.I., Plasterk, R.H., and Sternberg, P.W. (1995). Participation of the protein Go in multiple aspects of behavior in C. elegans. Science 267, $1652-1655$. Abstract

Peden, E.M., and Barr, M.M. (2005). The KLP-6 Kinesin Is Required for Male Mating Behaviors and Polycystin Localization in Caenorhabditis elegans. Curr. Biol. 15, 394-404. Abstract Article

Qin, H., Rosenbaum, J.L., and Barr, M.M. (2001). An autosomal recessive polycystic kidney disease gene homolog is involved in intraflagellar transport in C. elegans ciliated sensory neurons. Curr. Biol. 11, 457-461. Abstract Article

Simon, J.M., and Sternberg, P.W. (2002). Evidence of a mate-finding cue in the hermaphrodite nematode Caenorhabditis elegans. Proc. Natl. Acad. Sci. USA 99, 1598-1603. Abstract Article

Sulston, J.E., Albertson, D.G., and Thomson, J.N. (1980). The Caenorhabditis elegans male: postembryonic development of nongonadal structures. Dev. Biol. 78, 542-576. Abstract Article

Sulston, J.E., and Horvitz, H.R. (1977). Post-embryonic cell lineages of the nematode, Caenorhabditis elegans. Dev. Biol. 56, 110-156. Abstract Article 
White, J. (1988). The anatomy. In The Nematode Caenorhabditis elegans, W.B. Wood and the Community of $C$. elegans Researchers. Wood, ed. (Cold Spring Harbor, NY, Cold Spring Harbor Laboratory Press), pp. 81-122.

White, J.G., Southgate, E., Thomson, J.N., and Brenner, S. (1986). The structure of the nervous system of the nematode Caenorhabditis elegans: the mind of a worm. Phil. Trans. R. Soc. Lond. 314, 1-340.

All WormBook content, except where otherwise noted, is licensed under a Creative

Commons Attribution License. 